\title{
SOLVING THE PALLET LOADING PROBLEM MORE EFFICIENTLY
}

\author{
Josef Nelißen, RWTH Aachen
}

This paper looks at the packing problem called the 'pallet loading problem' (PLP for short) - that of finding an optimal layout for identical rectangular boxes on a rectangular pallet (a problem of type (3, B, O, C) according to the typology of Dyckhoff). Practical considerations usually require that the boxes must be placed orthogonally (i.e. with the box edges parallel to the pallet edges), and in layers in which the vertical orientation of the boxes is fixed. These restrictions mean that the problem is reduced to the two-dimensional problem of packing a large containing rectangle, orthogonally, with identical copies of a small 'contained' rectangle (type (2, B, O, C)). This problem is commonly referred to as pallet loading problem as well.

Since 1979 many heuristics have been developed to solve the PLP, mainly based on the placing of blocks, rectangles which contain a number of packing rectangles with equal orientation. The optimality of a solution can be proven by upper bounds, for whose computation elaborate procedures have been developed. If the heuristics fail to reach the upper bound, exact algorithms with an exponentially increasing running time have to be applied.

After an introduction the talk first presents a new method to compute upper bounds, which tries to reduce the problem to another problem the exact solution of which is known. Despite its simplicity, this method has been proven to be quite useful in cases where other piocedures fail.

In the second part the notion of partitions, a very important concept for the PLP, is introduced. Extending work done by Dowsland and Naujoks, this can on the one hand be used to characterize a set of equivalent instances of the PLP, which can be indexed by an open interval of the real line. On the other hand the partitions can be used to define a linear programming problem, whose solution with different cost functions leads to a deeper insight into the structure of an optimal solution. By means of a one-dimensional minimizer the first property can be used to find an instance of an equivalence class which has almost minimal waste. Structure analysis of this instance, based on the second property, often leads to a decreased upper bound.

In the third part a new heuristic is described, which reduces the given problem instance by placing L-shapes, formed of three blocks, in the lower left corner of the large rectangle, and solves the resulting instance either by block heuristics or an iteration of this L-reduction. This approach, which can be regarded as an extension of the diagonal heuristic of Exeler, often yields optimal solutions where block heuristics fail.

The last part describes an exact Branch\&Bound algorithm, which doesn't operate on the given instance of the problem, but on the above mentioned equivalent problem with minimal waste, which often results in a vast reduction of run time. By use of a grid technique, preprocessing, upper bounds on the number of vertical/horizontal placed packing rectangles, and upper and lower bounds on the frequency of partitions, the algorithm is able to solve medium sized problems in reasonable time. 